University of South Florida

DIGITAL COMMONS

Digital Commons @ University of

@ UNIVERSITY OF SOUTH FLORIDA

South Florida

Integrative Biology Faculty and Staff

Publications

Integrative Biology

$1-2009$

\title{
Are Morphological Specializations of the Hyolingual System in Chameleons and Salamanders Tuned to Demands on Performance?
}

\author{
Anthony Herrel \\ University of Antwerp, Belgium \\ Stephen M. Deban \\ University of South Florida, email@removeduser.com \\ Vicky Schaerlaeken \\ University of Antwerp, Belgium \\ Jean-Pierre Timmermans \\ University of Antwerp, Belgium \\ D. Adriaens \\ Ghent University, Belgium
}

Follow this and additional works at: https://digitalcommons.usf.edu/bin_facpub

Part of the Medical Sciences Commons

\section{Scholar Commons Citation}

Herrel, Anthony; Deban, Stephen M.; Schaerlaeken, Vicky; Timmermans, Jean-Pierre; and Adriaens, D., "Are Morphological Specializations of the Hyolingual System in Chameleons and Salamanders Tuned to Demands on Performance?" (2009). Integrative Biology Faculty and Staff Publications. 3.

https://digitalcommons.usf.edu/bin_facpub/3

This Conference Proceeding is brought to you for free and open access by the Integrative Biology at Digital Commons @ University of South Florida. It has been accepted for inclusion in Integrative Biology Faculty and Staff Publications by an authorized administrator of Digital Commons @ University of South Florida. For more information, please contact digitalcommons@usf.edu. 


\section{Are Morphological Specializations of the Hyolingual System in Chameleons and Salamanders Tuned to Demands on Performance?*}

\author{
Anthony Herrel ${ }^{1, \dagger}$ \\ Stephen M. Deban ${ }^{2}$ \\ Vicky Schaerlaeken ${ }^{1}$ \\ Jean-Pierre Timmermans ${ }^{3}$ \\ D. Adriaens ${ }^{4}$ \\ ${ }^{1}$ Department of Biology, University of Antwerp, \\ Universiteitsplein 1, B-2610 Antwerp, Belgium; ${ }^{2}$ Department \\ of Biology, SCA 110, 4202 East Fowler Avenue, University of \\ South Florida, Tampa, Florida 33620; ${ }^{3}$ Department of \\ Veterinary Sciences, University of Antwerp, \\ Groenenborgerlaan 171, B-2020 Antwerp, Belgium; \\ ${ }^{4}$ Department of Biology, Ghent University, K.L. \\ Ledeganckstraat 35, B-9000 Ghent, Belgium
}

Accepted 5/8/2008; Electronically Published 11/13/2008

\begin{abstract}
Extremely specialized and long tongues used for prey capture have evolved independently in plethodontid salamanders and chameleons. In both systems, the demands on tongue projection are probably similar: to maximize projection velocity and distance. Consequently, many of the design features of the projection system in these two groups have converged to an astonishing degree. Both involve the use of power amplification systems based on helically wound muscle fibers that load internal connective tissue sheets as illustrated in previous studies. Demands imposed on tongue retraction, however, are different to some degree. Although in both groups there is a clear demand for retraction capacity (given the long projection distances), in chameleons there is an added demand for force because they eat large and heavy prey. As indicated by our data, plethodontid salamanders have extremely long tongue retractors with normal striated muscle. Chameleons, on the other hand, evolved long retractors of the supercontracting type. Interestingly, our data show that at least in chameleons, the extreme design of the tongue in function of prey capture appears to have consequences on prey transport, resulting in an increased dependence on the hyoid. In turn, this has lead to an increase in transport-

\footnotetext{
* This paper was a contribution to the symposium "Functional Consequences of Extreme Adaptations of the Trophic Apparatus in Craniates," organized by Dominique Adriaens and Anthony Herrel, at the Eighth International Congress on Vertebrate Morphology, Paris, France, 2007.

${ }^{\dagger}$ Corresponding author; e-mail: anthony.herrel@ua.ac.be.
}

Physiological and Biochemical Zoology 82(1):29-39. 2009. (C) 2009 by The University of Chicago. All rights reserved. 1522-2152/2009/8201-8070\$15.00 DOI: $10.1086 / 589950$ cycle duration and an increase in the number of cycles needed to transport prey in comparison with closely related agamid lizards. Clearly, extreme morphological specializations are tuned to functional and ecological demands and may induce a reduced performance in other functions performed by the same set of integrated structures.

\section{Introduction}

Morphological specializations are often assumed to be the result of natural selection on morphology in response to specific functional or ecological demands. For example, the long beaks or snouts of nectar-eating birds and bats are thought to be the result of coevolution between plants and their pollinators (Muchhala 2007a, 2007b). Animals with longer beaks and snouts will have a performance advantage because they are able to sample a wider range of flowers, including those with the deepest corollas (Fleming et al. 2005; Muchhala 2006). Additionally, many bats, birds, and bumblebees have developed long tongues that allow them to reach into the deepest flowers without needing extremely elongated heads (Muchhala 2006).

The vertebrate tongue is an organ that is crucial to a wide variety of functions, including important roles in prey transport and swallowing, drinking, breathing, and even chemoreception and prey capture in some groups, such as lizards (Bels et al. 1994; Schwenk 2000; Herrel et al. 2001c). Thus, specialization of the tongue may be constrained by functional trade-offs imposed by the different functions. For example, specialization of the tongue for chemoreceptive purposes in many lizards and snakes constrains its use in prey capture and transport, and thus alternative capture (i.e., jaw prehension) and transport (e.g., inertial transport) strategies have been developed (Schwenk 2000). In other, animals such as anteaters (Redford 1985), many frogs (Nishikawa 1999, 2000), chameleons (Wainwright et al. 1991; Wainwright and Bennett 1992a, 1992b; Herrel et al. 2000), and plethodontid salamanders (Deban 1997; Deban et al. 1997; Wake and Deban 2000), the tongue has evolved into a highly specialized prey-capture organ.

If the tongue is used for prey capture, the functional demands imposed on it are clearly dependent on the distance of protrusion/projection and the size of prey that are captured. Tongue-extension length is probably relevant to predators because the further the tongue can be extended, the greater the number of prey that come within reach of the tongue without the need of predator movement, a feature especially crucial for cryptic sit-and-wait predators. Generally, two types of tongue extension are possible in vertebrates. Most commonly, the 
tongue functions as a muscular hydrostat. This mechanism depends on the active contraction of intrinsic muscles (Kier and Smith 1985; Smith 1988; Smith and Kier 1989). Although such a system has the advantage of being accurate because it allows for online modulation, it is also slow, and its extension is directly limited by muscle shortening (Nishikawa et al. 1999; Nishikawa 2000; Meyers et al. 2004). Alternatively, the tongue can be projected from the mouth and then lengthen under its own inertia. In ballistic systems, tongue extension is rapid but does not allow for modulation of the trajectory after release from the mouth. Tongue-extension length in such ballistic systems is probably limited by muscle stretch and the presence of connective tissue in the tongue (Peters and Nishikawa 1999; Nishikawa 2000). Additionally, tongue extension will be determined by the "takeoff" velocity of the tongue from the mouth (Hill 1950; Marsh 1994; Aerts 1998). Higher takeoff velocities are, in turn, dependent on the acceleration that can be imparted to the tongue, which is dictated by the ability of the musculoskeletal system to generate mechanical power (Lutz and Rome 1994; Aerts 1998; Askew and Marsh 2002; de Groot and van Leeuwen 2004; Lappin et al. 2006; Deban et al. 2007).

However, if the tongue is projected from the mouth to a great length, this will also impose demands on the tongueretraction system. Muscle shortening of typical cross-striated vertebrate muscle is limited to $50 \%$ of its resting length (Huxley 1965; Gordon et al. 1966). Thus, if ballistic projection involves extreme lengthening of the tongue as observed in chameleons, frogs, and plethodontid salamanders (Wainwright et al. 1991; Nishikawa 2000; Wake and Deban 2000), then the retractor muscles will probably have to be very long. This may, however, limit the functional capacity of the tongue retractor when the tongue is used during prey transport and swallowing (Herrel et al. 2001a, 2002). Near either end of its working range, the tongue retractor is faced with a decrease in its force-generating capacity because the muscle can be expected to be operating on the ascending and/or descending limb of its length-tension curve (i.e., decreased overlap between thick and thin filaments; Burkholder and Lieber 2001; Rome and Lindstedt 1997).

Here, we review the design of the tongue in two groups of vertebrates that have independently evolved extreme tongueprojection and prey-capture behaviors: plethodontid salamanders and chameleons (Deban et al. 1997; Herrel et al. 2000, $2001 b$, 2002). In doing so we aim to (1) explore whether functional demands imposed by the ecology and lifestyle of these animals have resulted in similar morphologies in these phylogenetically distantly related taxa and (2) investigate whether morphological specialization for extreme functional demands has limited the capacity of the tongue to function in other behaviors. In addition to reviewing the literature, we provide new data on the ultrastructure of the tongue retractors in $\mathrm{Hy}$ dromantes and previously unpublished data on the use of the hyoid during prey transport in an agamid lizard (Pogona vitticeps) with a generalized tongue morphology compared with chameleons (Chamaeleo calyptratus and Furcifer oustaleti).

\section{Material and Methods}

Histology

The morphology of the hyobranchial system of Hydromantes italicus, Salamandra salamandra, Laudakia stellio, and Chamaeleo calyptratus was studied by serial cross sections. Laudakia stellio (an agamid lizard) and S. salamandra (a nonplethodontid salamander) were chosen for comparison because they are both nonspecialized but use their tongues for prey capture. Specimens were killed by an overdose of anesthetic (MS222 for the salamanders, Ketamine for the lizards), fixed in an $8 \%$ buffered formaldehyde solution, decalcified with Decalc $25 \%$ (HistoLab), dehydrated through an alcohol series, and embedded in Technovit 7100 (Kulzer-Heraus; C. calyptratus and $H$. italicus) or paraffin (L. stellio, S. salamandra). Series of semithin sections $(2-5 \mu \mathrm{m})$ were cut using a Leica Polycut SM 2500, stained with a nonspecific toluidine blue (C. calyptratus) or tissue specific modified trichrome stain ( S. salamandra, L. stellio, and $H$. italicus), and mounted with DPX. Images of the sections were obtained using a digital camera (Colorview 8, Soft Imaging System) mounted on a Polyvar-Reichert light microscope and processed with Analysis Docu (Soft Imaging System, ver. 3.0).

\section{Transmission Electron Microscopy}

For transmission electron microscopy (TEM), the tongue retractors of an adult $H$. italicus (snout-vent length [SVL] 50 $\mathrm{mm}$ ) were removed unilaterally. Tissue samples were removed from both the anterior and posterior parts of the muscle. Several small blocks of tissue taken from the middle part of the muscle were cut from the samples and thoroughly washed three times for $10 \mathrm{~min}$ in $0.1 \mathrm{~mol} \mathrm{~L}^{-1}$ sodium cacodylate buffer containing $7.5 \%$ saccharose and $0.05 \%$ calcium chloride. Next, the tissue was postfixed for $2 \mathrm{~h}$ at $4^{\circ} \mathrm{C}$ in $1 \%$ osmium tetroxide in $0.033 \mathrm{~mol} \mathrm{~L}^{-1}$ veronalacetate buffer containing $4 \%$ saccharose $(\mathrm{pH} 7.4)$. After postfixation, samples were rinsed three times for $10 \mathrm{~min}$ in $0.05 \mathrm{~mol} \mathrm{~L}^{-1}$ veronalacetate buffer containing $6 \%$ saccharose sodium cacodylate buffer $(\mathrm{pH} 7.4)$ and dehydrated through a graded series of ethanols. The samples were then embedded in Durcupan. Suitable longitudinal areas and cross sections of the striated muscle were selected on toluidine blue-stained semithin sections. Subsequently, ultrathin sections were stained with lead citrate and uranyl acetate and examined in a Philips CM10 electron microscope. Photographs were taken of all samples at different locations for several sections within each sample. Pictures were taken of both anteriorly and posteriorly located samples. The sarcomere length, filament lengths (thick and thin), and I-band thickness were measured for 30 sarcomeres using TPSdig software (Rohlf 2004).

\section{Kinematics and Feeding Behavior}

Lizards (Pogona vitticeps, $N=2$; C. calyptratus, $N=1$; Furcifer oustaleti, $N=2$ ) used for feeding trials were obtained from a commercial dealer and transferred to the lab at the University 

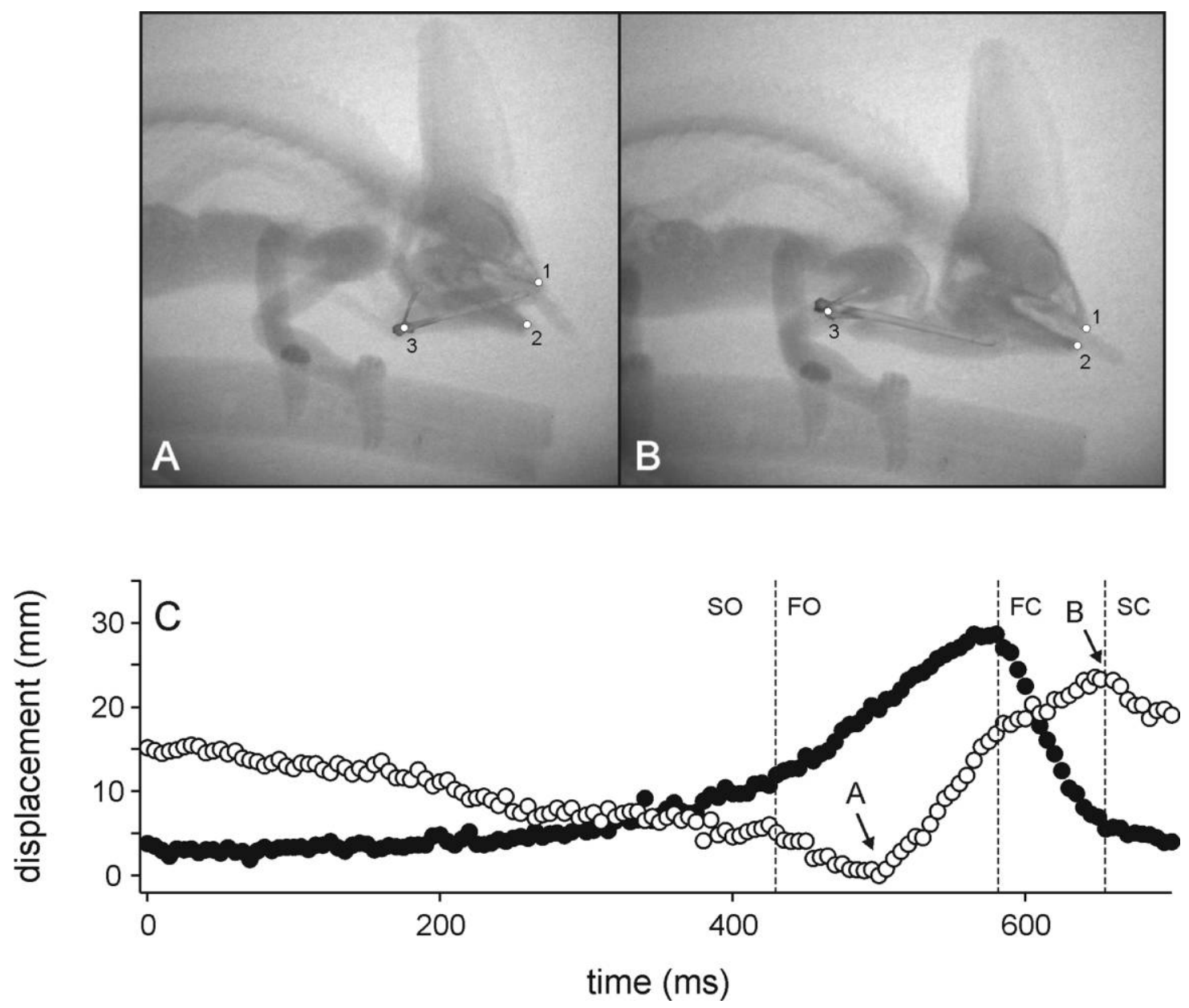

Figure 1. A, Picture of the hyoid in Camaeleo calyptratus in its most protracted position achieved during prey transport superimposed on a frame of an x-ray recording. $B$, The hyoid in its most retracted position. $C$, Displacement profiles of the jaws (filled circles) and the hyoid (open circles) during the transport of a large grasshopper in $C$. calyptratus. Arrows indicate the positions of points where the hyoid is in its most protracted $(A)$ and retracted $(B)$ position. White circles in $A$ and $B$ indicate the landmarks digitized on each frame: 1 , tip of upper jaw; 2, tip of lower jaw; 3 , base of the ceratobranchial. $S O$, slow opening phase; $F O$, fast opening phase; $F C$, fast closing phase; $S C$, slow closing phase.

of Antwerp. Animals were of similar size, the chameleons having an average lower-jaw length of $36.96 \pm 1.25 \mathrm{~mm}$ and the Pogona having an average lower-jaw length of $34.96 \pm 1.37$ $\mathrm{mm}$. The animals were kept in a vivarium on a $12 \mathrm{~L}: 12 \mathrm{D}$ cycle and were offered crickets, grasshoppers, and waxworms ad lib. The environmental temperature varied from $34^{\circ} \mathrm{C}$ during daytime to $22^{\circ} \mathrm{C}$ at night. An incandescent bulb provided the animals with a basking place at higher temperature.

\section{Video and Cineradiographic Recordings}

Pogona vitticeps and C. calyptratus were filmed in lateral view at $25 \mathrm{~Hz}$ using a handheld digital camera (Sony DCR-HC22E). The animals were offered large grasshoppers $(4 \mathrm{~cm})$. Simultaneously, high-speed x-ray videos were recorded using a Philips Optimus $x$-ray generator coupled to a 14-inch image intensifier with two zoom modes (10 and 6 inch) and a Redlake Motion Pro 2000 camera $(1,248 \times 1,024$ pixels $)$. Recordings of prey transport were made in lateral view using the 6-inch zoom function at 250 frames per second for P. vitticeps and 200 frames per second for C. calyptratus. All experiments were approved by the Animal Care and Use Committee at the University of Antwerp.

\section{Analyses}

Video recordings were reviewed using Midas Player software (Redlake, San Diego, CA, ver. 2.1.7). Only feeding sequences in which all the transport cycles of one feeding sequence were present were retained for further analysis. Based on these recordings, the number of transport cycles needed to transport a grasshopper and the average duration of a transport cycle were calculated. Cineradiographic recordings were reviewed using Midas Player software (ver. 2.1.7). Only feeding sequences in which all phases of a transport cycle ( $\mathrm{SO}$, slow opening; FO, fast opening; FC, fast closing; SC, slow closing; see Fig. 1) were present were used in further analysis. Three landmarks were digitized using Didge (Image Digitizing Software, Alistair Cul- 
A

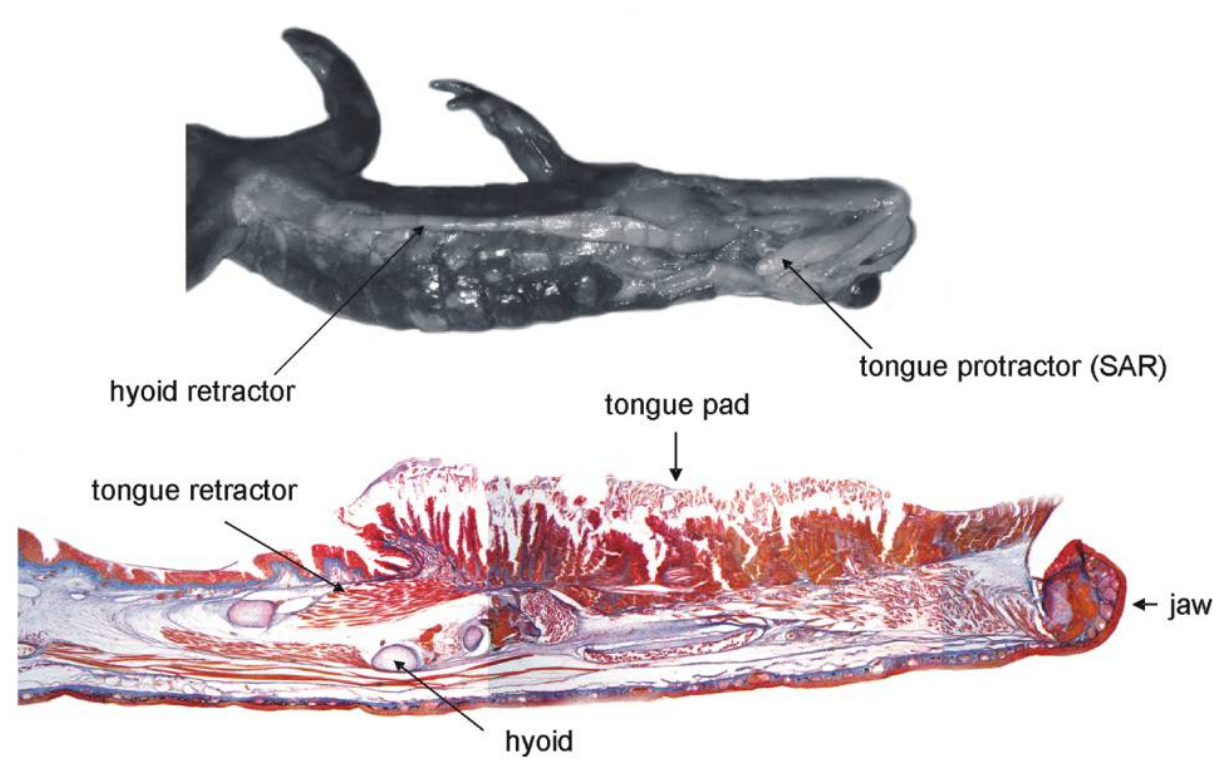

B

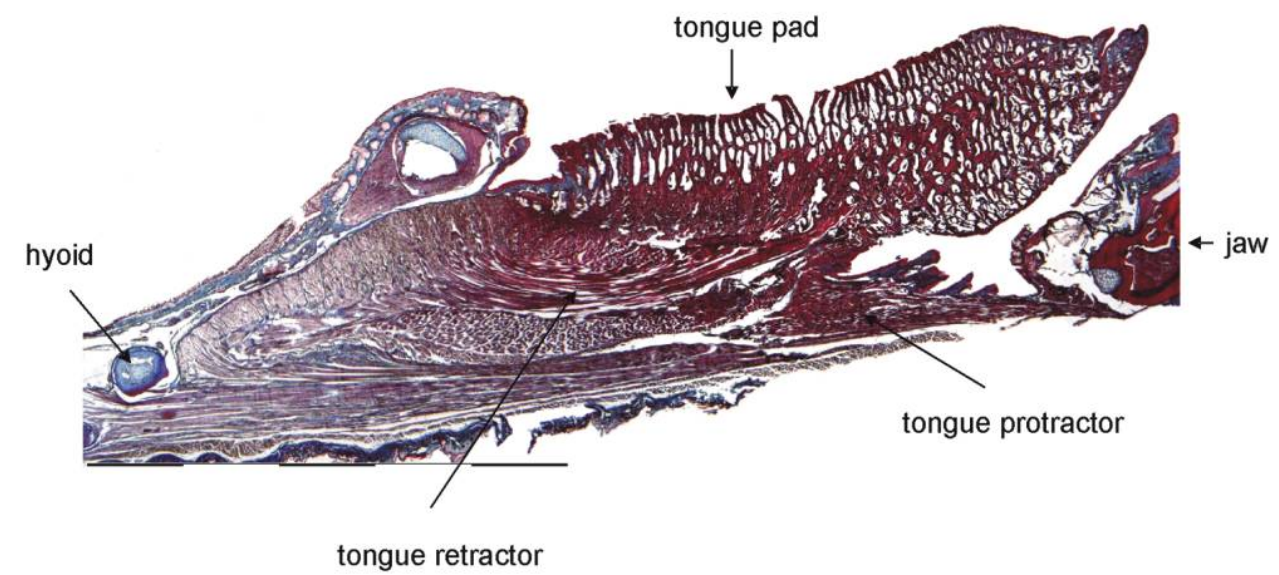

Figure 2. A, Top, ventrolateral view on the hyolingual system in Salamandra salamandra; bottom, saggital section through the tongue of an $S$. salamandra. B, saggital section through the tongue of Laudakia stellio (modified after Herrel et al. 2005). In the stains, muscle and bone are colored red/orange, and connective tissue and cartilage are colored blue.

lum, ver. 2.2.0). These landmarks included the anterior tip of the upper and lower jaw and base of the first ceratobranchial (Fig. 1). Based on $x-y$ coordinates of these markers, gape distance (distance between the jaw tips) and the displacement of the hyoid relative to the jaw during prey transport were calculated. Sixty intraoral transport cycles from seven different feeding sequences were analyzed for C. calyptratus, and 27 cycles from three feeding sequences for the first individual and 20 cycles from four feeding sequences for the second individual of F. oustaleti were analyzed. A total of 16 intraoral transport cycles from five complete feeding sequences were analyzed for two $P$. vitticeps.
Nested ANOVA with individual nested within group (agamids vs. chameleons) were used to test for differences in prey-transport kinematics between Pogona and the chameleons used in this study. Note that cycle-to-cycle and among-sequence variation is incorporated in the total individual variation and was not explicitly introduced as a factor in our analyses.

\section{Results and Discussion}

\section{Salamanders}

The tongue skeleton of terrestrial salamanders is composed of a series of articulating, mostly cartilaginous elements (Wake 
A
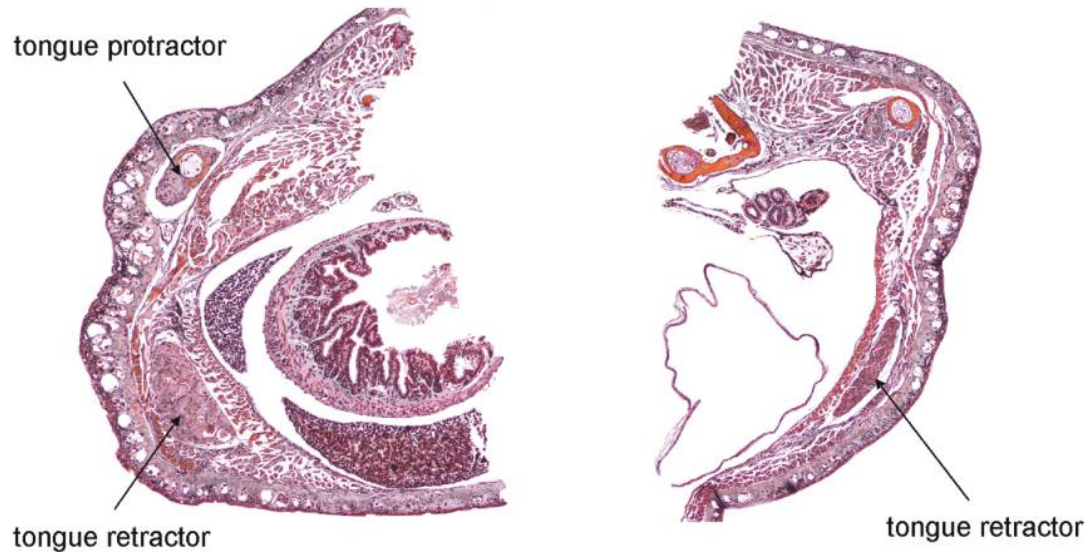

$\mathrm{XS}$ behind pectoral girdle

XS anterior to pelvic girdle

B

hyoid

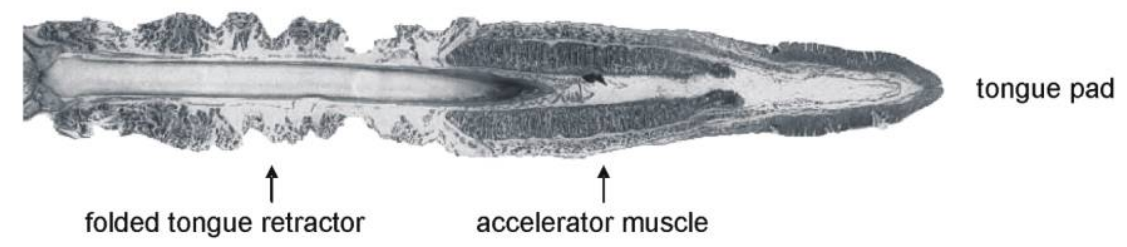

Figure 3. A, Left, cross section through the body (one half only) just posterior to the pectoral girdle in Hydromantes; right, cross section through the body (one half only) just anterior to the pectoral girdle in Hydromantes. Bone is colored orange, and muscle is colored pink. B, Frontal section through the hyolingual apparatus of Chameleo jacksonii (modified after Herrel et al. 2001a).

and Deban 2000). The basibranchial lies medially and provides support for the tongue pad. On each side, a first and second ceratobranchial articulate with the basibranchial, and an epibranchial articulates with the caudal ends of the first and second ceratobranchials (Lombard and Wake 1977; Wake and Deban 2000; Deban and Dicke 2004). The epibranchial in Hydromantes is greatly elongated in comparison with that of Salamandra (Lombard and Wake 1977; Wake and Deban 2000). During tongue protrusion/projection, the tongue skeleton folds medially as it is pulled and pushed forward. Whereas in Hydromantes, the tongue skeleton is free and can be projected completely from the mouth, in Salamandra, the posterior part of the tongue skeleton does not leave the mouth.

The $\mathrm{m}$. subarcualis rectus is the primary tongue-protractor muscle (Figs. 2, 3). It originates broadly along the edge of the ceratohyal and runs posteriorly to the epibranchial. The rostral portion has a fiber orientation that can pull the tongue skeleton forward. The caudal portion forms an elongated sheath around the epibranchial with a helically wound segmented arrangement of short muscle fibers and is active on average 107-117 ms before tongue projection in the plethodontids Bolitoglossa dofleini and Hydromantes imperialis (Deban and Dicke 2004; Deban et al. 2007; Fig. 4). In Salamandra, the m. subarcualis rectus does not form a sheath around the epibranchial but rather inserts along the posteroventral third of it. During tongue protrusion in Salamandra, the m. subarcualis rectus is active and pulls the epibranchial forward (Dockx and De Vree 1986; F. De Vree, personal communication). In Hydromantes, connective tissue sheets are present within the $\mathrm{m}$. subarcualis rectus posterior and between the muscle and the epibranchial, which are presumed to be deformed on contraction of the muscle and may thus have the potential to store elastic strain energy (Deban et al. 2007; Fig. 4). Tongue retraction in salamanders is accomplished by the $\mathrm{m}$. rectus cervicis posterior, which is a long straplike muscle that originates on the pelvis and inserts into the tongue pad (Deban and Dicke 1999; Figs. 2, 3). In Hydromantes species, a portion of the m. rectus cervicis posterior lies in a loop in the throat just in front of the heart when the tongue is at rest (Lombard and Wake 1977; Deban et al. 1997). In both Salamandra and Hydromantes, the muscle is activated before prey contact and remains active throughout tongue retraction (Dockx and De Vree 1986; Deban and Dicke 1999; F. De Vree, personal communication).

TEM data of the m. rectus cervicis collected specifically for this study show that the muscle is composed of typical crossstriated vertebrate muscle (Fig. 5). Neither anterior nor pos- 
A
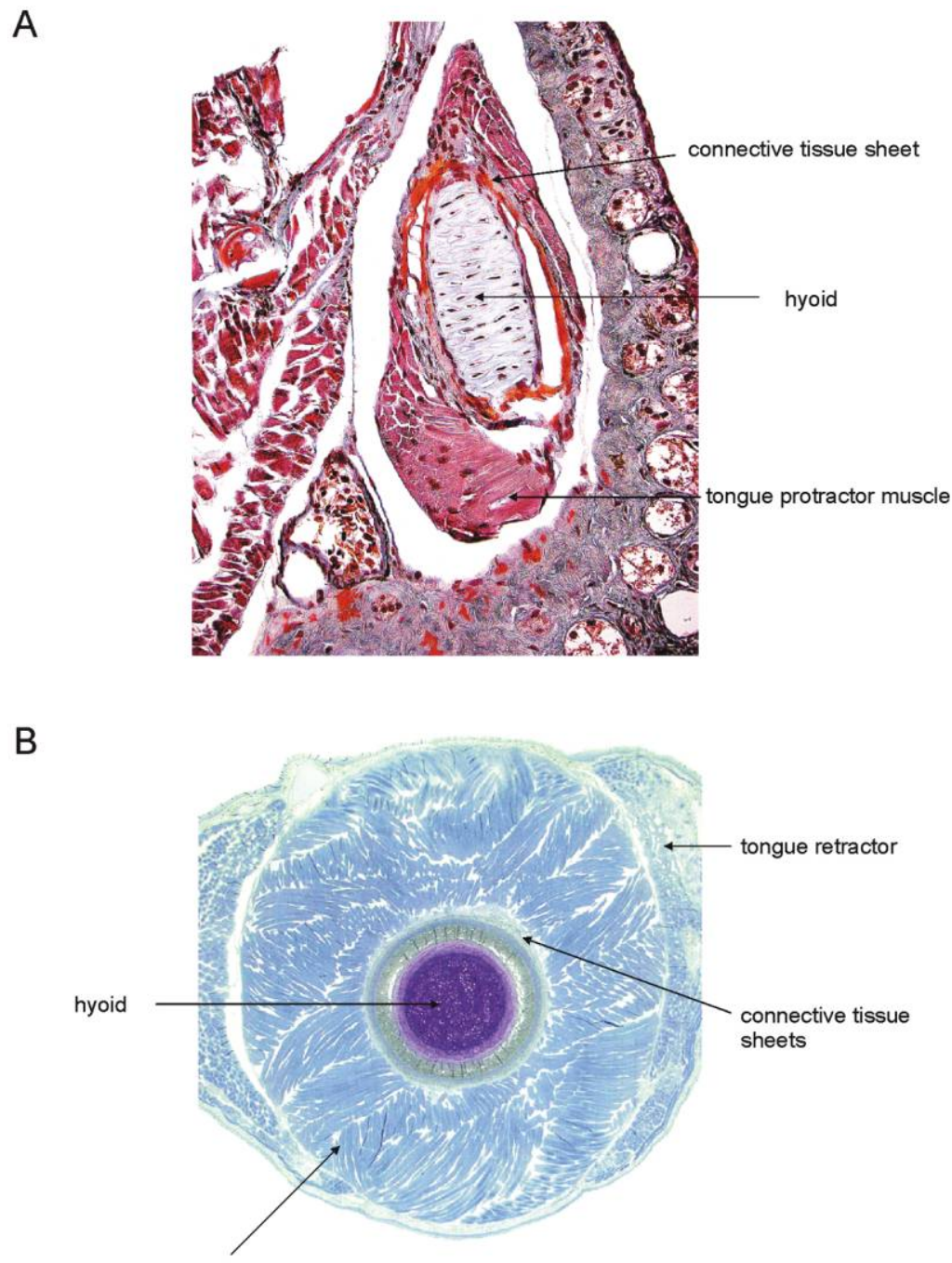

helical arrangement of muscle fibres

Figure 4. A, Cross section through the hyolingual apparatus in Hydromantes italicus at the level of the pectoral girdle. Note the cartilagenous epibranchial (blue) surrounded by connective tissue (orange) and muscle (pink). B. Cross section through the hyolingual system in Chameleo calyptratus at mid tongue. Note the dense centrally positioned cartilage of the entoglossal process surrounded by connective tissue sheets and the helically wound accelerator muscle.

terior samples show supercontracting muscle fibers. Sarcomere lengths are slightly shorter than typical vertebrate sarcomeres $(1.74 \pm 0.06 \mu \mathrm{m}$; Table 1$)$ with fairly typical I bands $(0.33 \pm$ $0.06 \mu \mathrm{m})$. Filament lengths also do not deviate greatly from the typical vertebrate pattern (thick: $1.41 \pm 0.02 \mu \mathrm{m}$; thin: $0.81 \pm 0.03 \mu \mathrm{m})$. Interestingly, the length of the tongue retractor of Hydromantes is slightly longer than the tongueprotrusion length ( $80 \%$ of body length; see Table 1$)$, suggesting that it can indeed operate using a typical vertebrate skeletalmuscle sarcomere structure contracting to approximately $50 \%$ of resting length. However, near full extension and retraction, the force output of the muscle is probably reduced.

\section{Lizards}

The tongue skeleton of the lizards examined here consists of a centrally positioned entoglossal process and three pairs of ar- ticulating elements in Pogona but only two pairs in Chameleo. Chameleons differ from agamid lizards by the presence of an elongated and parallel-sided entoglossal process, the absence of the second pair of ceratobranchials, a reduction of the length of the ceratohyals, and an anteriodorsad rotation of the first pair of ceratobranchials (see Herrel et al. 2001b). During tongue protrusion, the tongue skeleton is protracted, and the ceratobranchials are folded backward by the combined action of the mandibulohyoideus and sternohyoideus muscles (Wainwright and Bennett 1992a, 1992b; Meyers and Nishikawa 2000). In agamid lizards, the hyoid is also protracted, but to a lesser degree (Schwenk 2000). Neither in chameleons nor in agamids does the tongue skeleton leave the mouth entirely on tongue protrusion/projection.

The main tongue-protractor muscles in agamid lizards consist of the paired lateral, medial, and internal mm. genioglossi, 


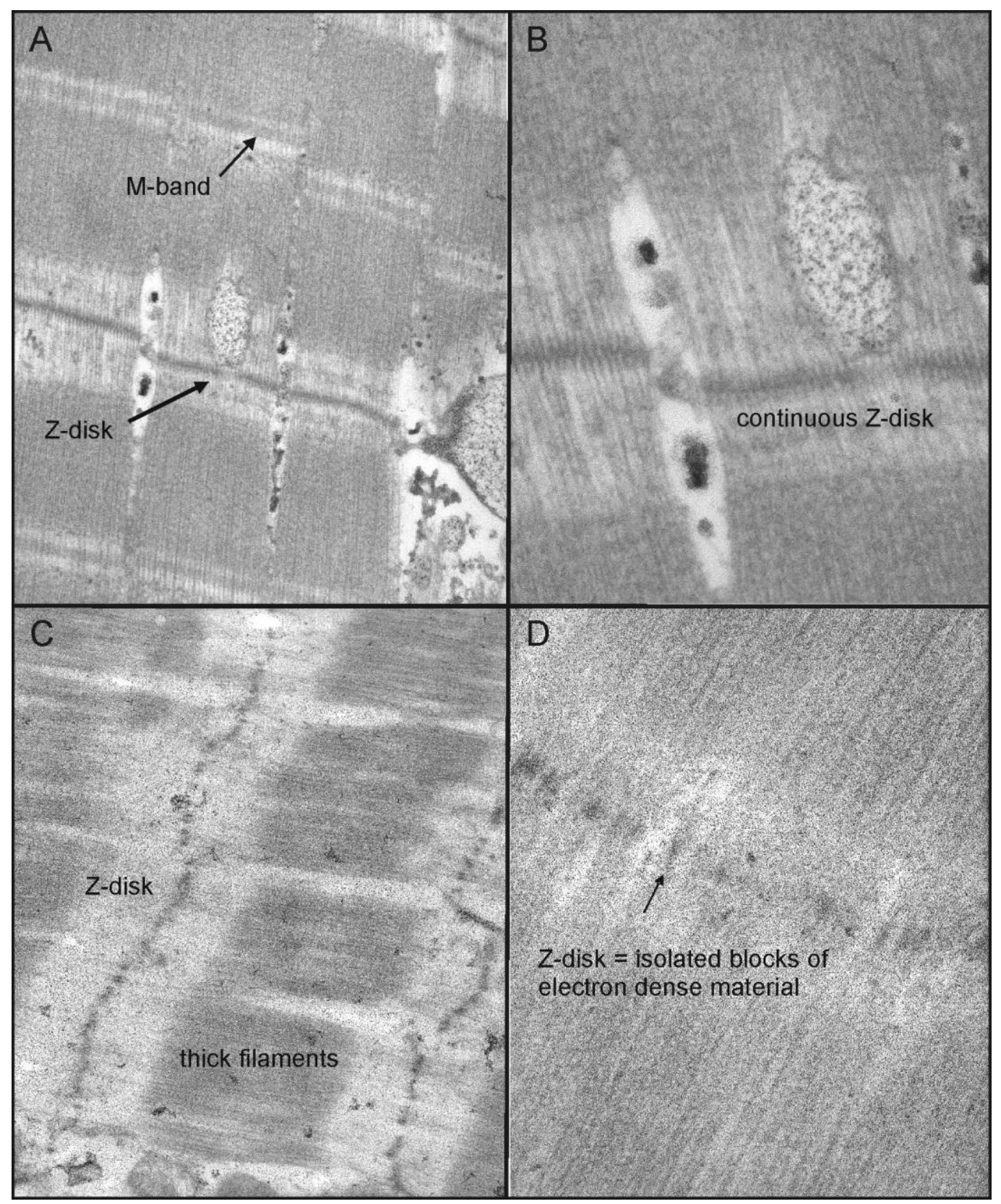

Figure 5. A, Transmission electron micrographs (TEMs) through the tongue-retractor muscle in Hydromantes italicus. $B$, detail of $A$ showing the continuous Z-disk structure. C, TEMs through the tongue-retractor muscle in Chameleo calyptratus. D, Detail showing how the $\mathrm{Z}$ disk is composed of isolated blocks of electron-dense material.

which run from the mandible to the tongue (Fig. 2; Herrel et al. 1995; Meyers and Nishikawa 2000). Additionally, the socalled ring muscle causes forward sliding of the tongue on the tapered entoglossal process (Smith 1988; Herrel et al. 1995). Hyoid protraction is achieved by the action of the paired mandibulohyoideus muscles (Herrel et al. 1995; Meyers and Nishikawa 2000). Tongue retraction is achieved by activity of the $\mathrm{m}$. hyoglossus, which runs from the first ceratobranchial into the tongue. Simultaneously, the hyoid is retracted by the paired hyoid retactors ( $\mathrm{m}$. sternohyoideus superficialis and profundus, $\mathrm{m}$. omohyoideus), which run from the pectoral girdle to the hyobranchium (Herrel et al. 1995; Meyers and Nishikawa 2000). The major difference in chameleons compared with agamid lizards is the shift in insertion of the $\mathrm{mm}$. genioglossi from the tongue to the floor of the mouth (Herrel et al. 2001b). Thus, tongue protrusion/projection in chameleons is achieved solely by the activity of the m. accelerator (the homologue to the ring muscle in agamids; see Wainwright and Bennett 1992a, $1992 b$ ). Protrusion of the hyobranchium in chameleons is achieved by the $\mathrm{mm}$. mandibulohyoidei, as is the case in agamids (Wainwright and Bennett 1992a; Herrel et al. 1995). The m. accelerator consists of helically wound short muscle 
Table 1: Summary of morphological and functional attributes of the hyolingual system in a typical plethodontid salamander (Hydromantes italicus) and a chameleon (Chameleo calyptratus)

\begin{tabular}{lcc}
\hline & Hydromantes italicus & Chameleo calyptratus \\
\hline Body mass $(\mathrm{g})$ & 5 & 100 \\
Snout-vent length $(\mathrm{mm})$ & 50 & 150 \\
Tongue length $(\mathrm{mm})$ & 3.6 & 17 \\
Tongue-extension length $(\mathrm{mm})$ & 39.2 & 315 \\
Tongue-extension duration $(\mathrm{ms})$ & 14 & 30 \\
Tongue-retractor length $(\mathrm{mm})$ & 34.5 & 41 \\
Tongue-retractor sarcomere length $(\mu \mathrm{m})$ & $1.74 \pm .06$ & $2.28 \pm .1$ \\
Tongue-retractor Z-disk perforations & No & Yes \\
Maximal prey size (\% body mass) & $3^{\text {a }}$ & $30^{\mathrm{b}}$ \\
\hline$\quad$ Note. Table entries are based on one adult individual of each species. Sarcomere lengths were determined for \\
30 sarcomeres in the salamander and 15 sarcomeres in the chameleon. \\
${ }^{\mathrm{a}}$ Based on Vignoli et al. 2006. \\
${ }^{\mathrm{b}}$ Personal observation (by A. Herrel) of the maximal prey size the C. calyptratus individuals were willing to \\
eat.
\end{tabular}

fibers surrounding the entoglossal process (Figs. 3, 4; Herrel et al. 2001b; de Groot and van Leeuwen 2004). Between the accelerator muscle and the entoglossal process, there are several layers of connective tissue sheets present (de Groot and van Leeuwen 2004). The accelerator muscle is activated 200-300 ms before the onset of tongue projection; during this time, these collagenous sheets are hypothesized to strain and store elastic energy for the tongue launch (de Groot and Van Leeuwen 2004).

A review of the literature shows that the tongue retractor in chameleons is unusual, as it is composed of supercontracting muscle fibers characterized by perforations in the $\mathrm{Z}$ disks (Fig. 5; Rice 1973; Herrel et al. 2001a, 2002). It is complexly folded in rest (Fig. 3), and its length is about half that of the body (Table 1). However, on projection, the muscle can be stretched up to four times its resting length, given that tongue-projection distances of up to two body lengths are not uncommon in many chameleons (Wainwright et al. 1991; A. Herrel, personal observation).

\section{Convergence in Morphology and Design}

The degree of convergence in the tongue-projection system in chameleons and plethodontid salamanders is striking. Independently, both groups have evolved similar tongue-projection systems relying on power amplification. Given similar functional demands but a radically divergent Bauplan, evolution has resulted in a remarkably similar solution in the mechanics (power amplifier) and morphology (helically wound muscle surrounding cartilaginous structure with circular connective tissue sheets that are preloaded and can store elastic energy; see Fig. 4). The difference in the projection system lies in the details. Whereas the tongue-protractor muscle is shot out of the mouth in the chameleons, the muscle remains inside the body in the plethodontids, and the hyobranchium with adhering tongue is propelled from the mouth. Although both systems are clearly high-performance systems, salamanders appear to be generating substantially higher power outputs during tongue projection (de Groot and van Leeuwen 2004; Deban et al. 2007). However, this may be partially a scaling effect, because smaller animals are expected to produce higher power outputs (Hill 1950). Clearly, an analysis of tongue projection in juvenile chameleons or dwarf chameleons of the genus Brookesia or Rhampholeon could shed light on this.

Tongue retraction clearly involves different morphologies associated with the different mechanical demands. Whereas in chameleons, there is a demand for both force production (chameleons are known to eat relatively large prey; see Luiselli and Rugiero 1996; Pleguezuelos et al. 1999; Herrel et al. 2000; Keren-Rotem et al. 2006) and retraction capacity (the tongue can be extended up to $200 \%$ of body length), plethodontids eat relatively smaller prey (up to 3\% of body mass; see Lynch 1985; Larsen et al. 1989; Vignoli et al. 2006) and do not extend their tongues that far (Wake and Deban 2000). Consequently, different solutions are presented to the problems associated with tongue retraction in the two groups. In the plethodontids, the retraction system is characterized by an extremely elongated tongue-retractor muscle that attaches onto the pelvis and, in rest, lies in a loop in front of the heart (Deban et al. 1997). Tongue retraction is relatively rapid compared with chameleons, sometimes taking as little as $30 \mathrm{~ms}$. In chameleons, on the other hand, the muscle is also relatively long but is characterized by the presence of supercontracting muscle fibers that allow cross-bridge cycling and thus force production beyond the limits of typical cross-striated vertebrate muscle (Rice 1973; Herrel et al. 2001a, 2002). Tongue retraction, however, is much slower in chameleons ( $\pm 300 \mathrm{~ms}$; Wainwright et al. 1991).

Despite the differences mentioned above, there are also a number of similarities in the retraction systems of both groups. Clearly, both plethodontid salamanders and chameleons have evolved long retractor muscles that are folded at rest and have broad length-tension curves. Because the tongue retractors need 
Table 2: Summary kinematics of the transport of grasshoppers in Pogona vitticeps and Chameleo calyptratus

\begin{tabular}{lccc}
\hline & $\begin{array}{c}\text { Average Number of } \\
\text { Transport Cycles }\end{array}$ & $\begin{array}{l}\text { Average Transport } \\
\text { Cycle Duration }(\mathrm{s})\end{array}$ & $\begin{array}{l}\text { Average Hyoid } \\
\text { Displacement }(\mathrm{mm})\end{array}$ \\
\hline Pogona vitticeps $(N=2)$ & $12.29 \pm 5.02$ & $.46 \pm .09$ & $5.87 \pm 2.41$ \\
Chameleo calyptratus $(N=3)$ & $20.26 \pm 7.28$ & $1.47 \pm .36$ & $12.79 \pm 3.35$ \\
\hline
\end{tabular}

to generate force throughout tongue retraction, in chameleons, they developed supercontracting sarcomeres. Plethodontids, on the other hand, benefited from the design of generalized salamanders, as illustrated by Salamandra; these animals also have a long tongue-retractor muscle inserting at the pelvic girdle. Thus, by increasing the length of the tongue retractors to a relatively minor extent, they were able to retract their tongues after projection and sustained only a small cost in retraction speed. Chameleons have strong tongue retractors due to the supercontractile properties of the muscle but are also 10 times slower than salamanders. Clearly, the constraints imposed by Bauplan and ecology define the morphological solutions in light of the mechanical demands imposed.

Interestingly, however, on complete retraction of the tongue into the mouth, the tongue-retractor muscle in chameleons is no longer able to generate much force (Herrel et al. 2001a, 2002). Although the overall prey-transport kinematics are similar in chameleons and agamid lizards (So et al. 1992; Herrel et al. 1996), the hyoid rather than the tongue is recruited as the principal element ensuring prey transport and repositioning in chameleons as suggested by our data. Indeed, movements of the hyoid in chameleons are more extensive than in Pogona vitticeps $\left(F_{1,3.61}=9.955 ; P=0.040\right)$ while transporting identical prey (Table 2). Moreover, chameleons tend to use more transport cycles on average $\left(F_{1,3.45}=5.79 ; P=0.08\right)$, and each individual transport cycle takes significantly longer compared with Pogona $\left(F_{1,5.13}=72.25 ; P<0.001\right)$. Interestingly, on complete retraction, the hyoid nearly touches the sternum (Fig. 1), and consequently the sternohyoideus muscle that runs from the sternum to the back of the basihyal must be contracting to more than $50 \%$ of its resting length. Although this suggests that this muscle may also be of the supercontracting type, an analysis of the ultrastructure of the $\mathrm{m}$. sternohyoideus is needed to test this hypothesis. Plethodontid salamanders are probably faced with a similar problem because in rest, the tongueretractor muscle is slack and lies in a loop in front of the heart. Preliminary electromyographic data do suggest that the muscle is active during intraoral transport (S. Deban, personal observation), but unfortunately, little is known about the role of the tongue during prey transport in these animals. Future experiments involving sonomicrometry or cineradiography may help elucidate this further.

An extreme specialization in function of the tongue in prey capture in chameleons and salamanders may obviously have consequences for other functions performed by the same system. Although these negative effects could potentially constrain the evolution of these extreme morphologies and behaviors, plethodontid salamanders have partly overcome these constraints by decoupling at least one function (respiration) from the tongue. Whether the specialization of the tongue affects the efficiency of prey transport, however, remains unknown. In the chameleons, the role of the tongue during prey transport has been shifted to the hyoid apparatus. However, this shift comes at a cost as evidenced by both the larger number of transport cycles needed to transport a prey and the greater transportcycle duration in comparison with a closely related lizard with a nonspecialized tongue, as suggested by our data. However, given the lifestyle of chameleons, characterized by cryptic colors and an extreme sit-and-wait foraging strategy, the incurred fitness cost of an increased prey-transport duration is probably minimal.

In summary, our review and data suggest extensive convergence in two distantly related taxa faced with similar mechanical demands on the use of the tongue during prey capture. When functional demands differ as a result of differences in ecology (as illustrated in the case of tongue retraction), the design of the system differs as well. Thus, our data suggest that the morphology of the prey-capture system in chameleons and plethodontid salamanders is the result of an extensive coevolution of ecology, function, and behavior.

\section{Acknowledgments}

We thank B. De Kegel and J. Christiaens for their help with sectioning chameleons and salamanders and Francis Terloo for help with the transmission electron microscopy. The research of Vicky Schaerlaeken is funded by a PhD grant of the Institute for the Promotion of Innovation through Science and Technology in Flanders.

\section{Literature Cited}

Aerts P. 1998. Vertical jumping in Galago senegalensis: the quest for a hidden power amplifier. Philos Trans R Soc Lond B 353:1607-1620.

Askew G.N. and R.L. Marsh. 2002. Muscle designed for maximum short-term power output: quail flight muscle. J Exp Biol 205:2153-2160.

Bels V.L., M. Chardon, and K.V. Kardong. 1994. Biomechanics of the hyolingual system in Squamata. Pp. 197-240 in V.L. Bels, M. Chardon, and P. Vandewalle, eds. Advances in Com- 
parative and Environmental Physiology. Vol. 18. Springer, Berlin.

Burkholder T.J. and R.L. Lieber. 2001. Sarcomere length operating range of vertebrate muscles during movement. J Exp Biol 204:1529-1536.

Deban S.M. 1997. Modulation of prey-capture behavior in the plethodontid salamander Ensatina eschscholtzii. J Exp Biol 200:1951-1964.

Deban S.M. and U. Dicke. 1999. Motor control of tongue movement during prey capture in plethodontid salamanders. J Exp Biol 202:3699-3714.

. 2004. Activation patterns of the tongue-projector muscle during feeding in the imperial cave salamander, Hydromantes imperialis. J Exp Biol 207:2071-2081.

Deban S.M., J.C. O'Reilly, U. Dicke, and J.L. van Leeuwen. 2007. Extremely high-power tongue projection in plethodontid salamanders. J Exp Biol 210:655-667.

Deban S.M., D.B. Wake, and G. Roth. 1997. Salamander with a ballistic tongue. Nature 389:27-28.

de Groot J.H. and J.L. van Leeuwen. 2004. Evidence for an elastic projection mechanism in the chameleon tongue. Proc R Soc B 271:761-770.

Dockx P. and F. De Vree. 1986. Prey capture and intra-oral food transport in terrestrial salamanders. Stud Herpetol 1986:521-524.

Fleming T.H., N. Muchhala, and P. Ornelas. 2005. New world nectar-feeding vertebrates: community patterns and processes. Pp. 161-182 in V. Sanchez-Cordero and R.A. Medellín, eds. Contribuciones Mastozoológicos en Homenaje a Bernardo Villa-R. Instituto de Biología e Instituto de Ecología, UNAM, Mexico City.

Gordon A.M., A.F. Huxley, and F.J. Julian. 1966. The variation of isometric tension with sarcomere length in vertebrate muscle fibres. J Physiol 184:170-192.

Herrel A., M. Canbek, Ü. Özelmas, M. Uyanoglu, and M. Karakaya. 2005. Comparative functional analysis of the hyolingual anatomy in lacertid lizards. Anat Rec 284:561-573.

Herrel A., J. Cleuren, and F. De Vree. 1995. Prey capture in the lizard Agama stellio. J Morphol 224:313-329.

- 1996. Kinematics of feeding in the lizard Agama stellio. J Exp Biol 199:1727-1742

Herrel A., J.J. Meyers, P. Aerts, and K.C. Nishikawa. $2001 a$. Functional implications of supercontracting muscle in the chameleon tongue retractors. J Exp Biol 204:3621-3627.

Herrel A., J.J. Meyers, K.C. Nishikawa, and P. Aerts. 2000. The mechanics of prey prehension in chameleons. J Exp Biol 203: 3255-3263.

Herrel A., J.J. Meyers, K.C. Nishikawa, and F. De Vree. 2001 b. Morphology and histochemistry of the hyolingual apparatus in chameleons. J Morphol 249:154-170.

2001c. The evolution of feeding motor patterns in lizards: modulatory complexity and constraints. Am Zool 41: 1311-1320.

Herrel A., J.J. Meyers, J.-P. Timmermans, and K.C. Nishikawa. 2002. Supercontracting muscle: producing tension over extreme muscle lengths. J Exp Biol 205:2167-2173.
Hill A.V. 1950. The dimensions of animals and muscular dynamics. Sci Prog 38:209-230.

Huxley H.E. 1965. The mechanism of muscular contraction. Sci Am 213:18-27.

Keren-Rotem T., A. Bouskila, and E. Geffen. 2006. Ontogenetic habitat shift and risk of cannibalism in the common chameleon (Chamaeleo chamaeleon). Behav Ecol Sociobiol 59: 723-731.

Kier W.M. and K.K. Smith. 1985. Tongues, tentacles and trunks: the biomechanics and movement of muscular hydrostats. Zool J Linn Soc 83:207-324.

Lappin A.K., J.A. Monroy, J.Q. Pilarski, E.D. Zepnewski, D.J. Pierotti, and K.C. Nishikawa. 2006. Storage and recovery of elastic potential energy powers ballistic prey capture in toads. J Exp Biol 209:2535-2553.

Larsen J.H., Jr., J.T. Beneski Jr., and D.B. Wake. 1989. Hyolingual feeding systems of the Plethodontidae: comparative kinematics of prey capture by salamanders with free and attached tongues. J Exp Zool 252:25-33.

Lombard R.E. and D.B. Wake. 1977. Tongue evolution in the lungless salamanders, family Plethodontidae. II. Function and evolutionary diversity. J Morphol 153:39-80.

Luiselli L. and L. Rugiero. 1996. Chamaeleo chamaeleon (common chameleon) diet. Herpetol Rev 27:78-79.

Lutz G.J. and Rome L.C. 1994. Built for jumping: the design of the frog muscular system. Science 263:370-372.

Lynch J.F. 1985. The feeding ecology of Aneides flavipunctatus and sympatric plethodontid salamanders in northwestern California. J Herpetol 19:328-352.

Marsh R.L. 1994. Jumping ability of anuran amphibians. Pp. 51-111 in J.H. Jones, ed. Advances in Veterinary Science and Comparative Medicine: Comparative Vertebrate Exercise Physiology. Academic Press, New York.

Meyers J.J. and K.C. Nishikawa. 2000. Comparative study of tongue protrusion in three iguanian lizards: Sceloporus undulatus, Pseudotrapelus sinaitus and Chamaeleo jacksonii. J Exp Biol 203:2833-2849.

Meyers J.J., J.C. O’Reilly, J.M. Monroy, and K.C. Nishikawa. 2004. Mechanism of tongue protrusion in microhylid frogs. J Exp Biol 207:21-31.

Muchhala N. 2006. Nectar bat stows huge tongue in rib cage. Nature 444:701-702.

- 2007a. Adaptive trade-off in corolla shape mediates specialization for flowers pollinated by bats and hummingbirds. Am Nat 169:494-504.

2007b. Character displacement among bat-pollinated flowers of the genus Burmeistera: analysis of mechanism, process, and pattern. Proc R Soc B 274:2731-2737.

Nishikawa K.C. 1999. Neuromuscular control of prey capture in frogs. Philos Trans R Soc B 354:941-954.

- 2000. Feeding in frogs. Pp. 117-144 in K. Schwenk, ed. Feeding: Form, Function and Evolution in Tetrapod Vertebrates. Academic Press, San Diego, CA.

Nishikawa K.C., W.M. Kier, and K.K. Smith. 1999. Morphology and mechanics of tongue movement in the African pig-nosed 
frog (Hemisus marmoratum): a muscular hydrostatic model. J Exp Biol 202:771-780.

Peters S.E. and K.C. Nishikawa. 1999. Comparison of isometric contractile properties of the tongue muscles in three species of frogs, Litoria caerulea, Dyscophus guinetti and Bufo marinus. J Morphol 242:107-124.

Pleguezuelos J.M., J.C. Poveda, R. Monterrubio, and D. Ontiveros. 1999. Feeding habits of the common chameleon, Chamaeleo chamaeleon in the southeastern Iberian Peninsula. Isr J Zool 45:267-276.

Redford K.H. 1985. Feeding and food preference in captive and wild giant anteaters (Myrmecophaga tridactyla). J Zool (Lond) 205:559-572.

Rice M.J. 1973. Supercontracting muscle in a vertebrate. Nature 243:238-240.

Rohlf F.J. 2004. tpsDIG32. Version 1.40. http://life.bio.sunysb .edu/morph/index.html.

Rome L.C. and S.L. Lindstedt. 1997. Mechanical and metabolic design of the muscular system in vertebrates. Pp. 1587-1651 in W.H. Dantzler, ed. Handbook of Physiology. Section 13, vol. 2. Oxford University Press, New York.

Schwenk K. 2000. Feeding in lepidosaurs. Pp. 175-291 in K. Schwenk, ed. Feeding: Form, Function and Evolution in Tetrapod Vertebrates. Academic Press, San Diego, CA.

Smith K.K. 1988. Form and function of the tongue in agamid lizards with comments on its phylogenetic significance. J Morphol 196:157-171.

Smith K.K. and W.M. Kier. 1989. Trunks, tongues, and tentacles: moving with skeletons of muscle. Am Sci 77:28-35.

So K., P.C. Wainwright, and A.F. Bennett. 1992. Kinematics of prey processing in Chamaeleo jacksonii: conservation of function with morphological specialisation. J Zool (Lond) 226: $47-64$

Vignoli L., F. Caldera, and M.A. Bologna. 2006. Trophic niche of cave populations of Speleomantes italicus. J Nat Hist 40: $1841-1850$.

Wainwright P.C. and A.F. Bennett. 1992a. The mechanism of tongue projection in chameleons. I. Electromyographic tests of functional hypotheses. J Exp Biol 168:1-21.

- 1992b. The mechanism of tongue projection in chameleons. II. Role of shape change in a muscular hydrostat. J Exp Biol 168:23-40.

Wainwright P.C., D.M. Kraklau, and A.F. Bennett. 1991. Kinematics of the tongue projection in Chamaeleo oustaleti. J Exp Biol 159:109-133.

Wake D.B. and S.M. Deban. 2000. Terrestrial feeding in salamanders. Pp. 95-116 in K. Schwenk, ed. Feeding: Form, Function and Evolution in Tetrapod Vertebrates. Academic Press, San Diego, CA. 